\title{
A Realidade Aumentada em Situações de Aprendizagem na Educação Básica: Uma Revisão de Literatura
}

\author{
Sandro M. Rezende ${ }^{1}$, Juanice D. B. Gonçalves ${ }^{1}$, Sergio C. C. S. Pinto ${ }^{1}$, Cristina M. \\ C. Delou ${ }^{1}$ \\ ${ }^{1}$ Programa de Pós-graduação em Ciências, Tecnologias e Inclusão (PGCTIn) - \\ Universidade Federal Fluminense (UFF) - Niterói - RJ - Brasil \\ \{sandromiranda, judaumas, screspo, cristinadelou\}@id.uff.br
}

\begin{abstract}
Technological advances are increasingly present in various social spaces, including the school. In this work, we discuss the potential of technological resources in the teaching and learning process and, more specifically, we carry out a literature review in order to identify how learning situations involving augmented reality in Basic Education have been addressed in scientific productions in the last ten years. The results point to a series of possibilities involving the use of technologies such as smartphones, tablets and specific applications, especially in the development of locationbased games and inquiry-based learning.
\end{abstract}

Resumo. Os avanços tecnológicos estão cada vez mais presentes em diversos espaços sociais, entre eles o escolar. Neste trabalho, discutimos as potencialidades de recursos tecnológicos nos processos de ensino e aprendizagem e, mais especificamente, realizamos uma revisão de literatura com o objetivo de identificar como situações de aprendizagem envolvendo realidade aumentada na Educação Básica vêm sendo abordadas em produções científicas nos últimos dez anos. Os resultados apontam uma série de possibilidades envolvendo uso de tecnologias como smartphones, tablets e aplicativos especificos, especialmente no desenvolvimento de jogos baseados em localização e aprendizagem baseada em investigação.

\section{Introdução}

O uso de novas tecnologias de um modo geral tem sido amplamente disseminado na sociedade e observa-se que pessoas de diversas idades e grupos sociais utilizam de alguma forma recursos tecnológicos em sua vida, seja profissional ou pessoal. Da mesma forma, a presença das tecnologias digitais e interativas tem se tornado uma realidade cada vez mais presente no contexto educacional. Atualmente, a interatividade através de recursos digitais tem se apresentado como uma solução criativa em sala de aula, possibilitando a construção de espaços mais atrativos nas escolas e gerando maior interesse junto aos estudantes. Além disso, tais práticas pedagógicas possibilitam que os alunos se tornem protagonistas em seu processo de aprendizagem.

É urgente a discussão sobre a inserção de tecnologias digitais e interativas no ambiente educacional, tanto por serem inegáveis os benefícios que elas podem trazer aos alunos e professores, como por uma questão de acompanhar a evolução tecnológica global. Aproximar a educação e as tecnologias explorando os recursos de que as escolas dispõem faz parte do compromisso social e cultural da Educação com a sociedade 
informatizada, e contribui para o distanciamento de práticas de ensino engessadas e tradicionais.

Entre as tecnologias digitais interativas que vêm sendo utilizadas no contexto escolar encontram-se a realidade virtual, a realidade aumentada e os jogos digitais, ferramentas que apresentam diversas possibilidades de uso por estudantes e professores. Tais tecnologias ganham ainda maior destaque em um contexto em que os alunos nasceram e cresceram em uma sociedade altamente tecnológica, tendo recursos digitais como parte de seu cotidiano.

A realidade virtual é definida por [Tori, Kirner e Siscoutto 2006] como uma interface avançada para aplicações computacionais, permitindo a movimentação (navegação) e interação em tempo real, em um ambiente tridimensional, podendo fazer uso de dispositivos multissensoriais para atuação ou feedback. Esse recurso, quando aplicado em contextos educacionais, permite que o aluno não apenas interaja com uma situação de aprendizagem diferente como também possui o potencial de transportá-lo para uma realidade alternativa, com sensação da existência física do que está sendo experimentado.

Já a realidade aumentada consiste em uma tecnologia que combina o "mundo virtual" e o "mundo real", permitindo que o ambiente virtual seja transportado para o espaço real em tempo real [Silva 2013]. Segundo Forte e [Kirner 2009], "[...] a Realidade Aumentada trata do mundo real como ponto de partida para uma experiência que leva o utilizador a experimentar o mundo virtual". Nesse sentido, a realidade aumentada permite que o indivíduo interaja com o virtual de forma espontânea e realística, possibilitando que as informações sejam exploradas de maneira mais compreensível. O potencial de aplicabilidade e usabilidade da realidade aumentada é vasto e atualmente evidencia-se a sua utilização em diversas áreas de conhecimento, como na arquitetura, publicidade, design, educação e na geologia, entre outras áreas.

A realidade aumentada diferencia-se da realidade virtual, uma vez que esta possui o foco na imersão, e aquela consiste em uma combinação entre o "real" e o "virtual". Para [Azuma 2001], a realidade aumentada possui as seguintes propriedades: a combinação de objetos reais e virtuais em um mesmo ambiente real; a interatividade em tempo real; e registro de objetos reais e virtuais. Já [Macedo e Fernandes 2015] salientam que na utilização de situações de aprendizagem envolvendo realidade aumentada são observadas vantagens como a visualização do experimento em terceira dimensão, interação dos estudantes com esses experimentos, utilização prática e rápida a partir do momento em que o Objeto de Aprendizagem em RA foi elaborado, além de, em muitos casos, simplicidade e economia do equipamento utilizado.

Neste estudo, objetivamos lançar um olhar para a presença das tecnologias digitais e interativas no meio educacional e, mais especificamente, realizamos uma revisão bibliográfica acerca da construção de situações de aprendizagem baseadas em realidade aumentada na Educação Básica. O trabalho está dividido da seguinte forma: na seção 2, são apresentados os aspectos metodológicos da pesquisa; já na Seção 3, são apresentados os resultados das buscas, bem como são discutidos os principais aspectos observados no levantamento; e por fim, na seção 4 apresentamos as considerações finais. 


\section{Método}

A pesquisa realizada é um estudo exploratório que se caracteriza como qualitativo quanto à abordagem. O procedimento empregado foi a revisão de literatura, com o objetivo de identificar e analisar produções científicas nos últimos dez anos que abordem situações de aprendizagem envolvendo realidade aumentada na Educação Básica.

A questão de pesquisa foi definida da seguinte forma: o que as publicações científicas evidenciam sobre como artefatos voltados à realidade aumentada vêm sendo explorados em situações de ensino e aprendizagem na educação básica? Para a condução da revisão bibliográfica, foram realizadas buscas no portal de periódicos da CAPES e no Education Resources Information Center (ERIC), utilizando-se as expressões "realidade aumentada" AND "Educação Básica" e "augmented reality" AND "k-12 education".

Os critérios de inclusão de artigos para análise foram definidos da seguinte forma: i) o artigo aborda o uso da realidade aumentada na Educação Básica; ii) o artigo foi publicado nos últimos dez anos. Já os critérios de exclusão foram: i) o artigo aborda realidade aumentada, mas não apresenta situações de aprendizagem explorando artefatos voltados à realidade aumentada; ii) não é possível a visualização da versão completa do artigo.

\section{Resultados e discussão}

A partir das buscas obteve-se, inicialmente, um total de 126 artigos. Após leitura dos títulos e resumos, foram aplicados os critérios de inclusão e exclusão, tendo sido levantadas 10 publicações para análise. É importante mencionar que muitos trabalhos não foram incluídos no estudo pois, embora abordassem a temática da realidade aumentada, não apresentavam resultados que explorassem situações de aprendizagem envolvendo artefatos voltados à realidade aumentada. Os trabalhos selecionados são exibidos no Quadro 1.

Quadro 1. Artigos selecionados para análise

\begin{tabular}{|c|c|}
\hline Artigo & Autores e ano \\
\hline $\begin{array}{l}\text { [A1] Examining microscopic organisms under } \\
\text { augmented reality microscope: a } 5 \mathrm{E} \text { learning } \\
\text { model lesson }\end{array}$ & ABDUSSELAM, S. M. et al., 2018 \\
\hline $\begin{array}{l}\text { [A2] Augmented technology games: using } \\
\text { technology on a budget }\end{array}$ & ANNETTA, L. et al., 2012 \\
\hline $\begin{array}{c}\text { [A3] Investigating flow experience and scientific } \\
\text { practices during } \\
\text { a mobile serious educational game }\end{array}$ & $\begin{array}{l}\text { BRESSLER, D. M.; BODZIN, A. M., } \\
2016\end{array}$ \\
\hline $\begin{array}{l}\text { [A4] Using epistemic network analysis to examine } \\
\text { discourse } \\
\text { and scientific practice during a collaborative game }\end{array}$ & BRESSLER, D. M. et al., 2019 \\
\hline $\begin{array}{c}\text { [A5] Students' online interactive patterns in } \\
\text { augmented reality-based } \\
\text { inquiry activities }\end{array}$ & $\begin{array}{l}\text { CHIANG, T. H.C.; YANG, S. J. H.; } \\
\text { HWANG, G., } 2014\end{array}$ \\
\hline
\end{tabular}




\begin{tabular}{|c|c|}
\hline Artigo & Autores e ano \\
\hline $\begin{array}{c}\text { [A6] Active learning augmented reality for STEAM } \\
\text { education - a case study }\end{array}$ & $\begin{array}{c}\text { JESIONKOWSKA, J.; WILD, F.; } \\
\text { DEVAL, Y., 2020 }\end{array}$ \\
\hline $\begin{array}{c}\text { [A7] Engaging youth in computational thinking } \\
\text { practices through designing place-based mobile } \\
\text { games about local issues }\end{array}$ & $\begin{array}{c}\text { LITTS, B. K.; LEWIS, W. E.; } \\
\text { MORTENSEN, C. K., 2019 }\end{array}$ \\
\hline $\begin{array}{c}\text { [A8] Probability learning in mathematics using } \\
\text { augmented reality: } \\
\text { impact on student's learning gains and attitudes }\end{array}$ & CAI, S. et al., 2019 \\
\hline $\begin{array}{c}\text { [A9] Using Pokemon Go to teach integrative STEM } \\
\text { [A10] Augmented reality in education and training: } \\
\text { pedagogical }\end{array}$ & BARTHOLOMEW, S. R., 2017 \\
approaches and illustrative case studies & WANG, M. et al., 2017 \\
\hline
\end{tabular}

Todos os artigos selecionados foram lidos na íntegra, de modo que se pudesse analisar as situações de aprendizagem envolvendo realidade aumentada apresentadas nas publicações. Com o objetivo de responder à questão de pesquisa (o que as publicações científicas evidenciam sobre como artefatos voltados a realidade aumentada vêm sendo explorados em situações de ensino e aprendizagem na educação básica?), uma síntese dos objetivos e resultados dos estudos levantados é apresentada a seguir, bem como são apontados os modos como a realidade aumentada é abordada nos trabalhos.

[A1] Neste estudo, [Abdusselam et al. 2018], baseados em [Trowbridge, Bybee e Powell 2000, apud Abdusselam et al. 2018], propõem um modelo de aprendizagem 5E (Engagement, Exploration, Explanation, Elaboration, Evaluation), para estudantes entre 10 e 12 anos de idade. Nas atividades propostas, os participantes deveriam utilizar um microscópio de realidade aumentada para examinar organismos microscópicos, em uma metodologia de aprendizagem baseada em investigação (inquiry-based learning). Os autores concluíram que com a substituição do microscópio tradicional por uma tecnologia de realidade aumentada 3D em um laboratório de ciências, durante todas as etapas da atividade os estudantes tiveram a oportunidade de investigar e desenvolver habilidades de pensamento de alto nível, tendo sua motivação e interesses estimulados.

[A2] No estudo, [Annetta et al. 2012] apontam o aumento do uso de smartphones por estudantes entre 12 e 17 anos de idade, e descrevem como desenvolver jogos envolvendo realidade aumentada para o trabalho em sala de aula. Os autores se baseiam mais especificamente no aplicativo junaio, que permite que usuários utilizem instalações físicas dos arredores para criarem jogos de maneira rápida, sem a necessidade de habilidades de programação. Nessa perspectiva, os autores desenvolveram um jogo utilizando coordenadas GPS para marcar locais de interesse em diferentes espaços de uma escola. Assim, na medida em que os participantes exploravam o jogo, os mesmos eram levados a tais locais, nos quais podiam obter informações e realizar atividades que ativavam novas localizações a serem encontradas. Os autores concluíram que atividades como a descrita no trabalho não pretendem substituir outros tipos de instrução, mas sim possibilitam promover o engajamento de 
estudantes por meio de diferentes narrativas e novas metodologias como aprendizagem baseada em jogos e aprendizagem baseada em investigação.

[A3] Neste trabalho, [Bressler e Bodzin 2016] investigaram se um jogo educacional sério [Annetta 2008, apud Bressler e Bodzin 2016] era eficiente na promoção de práticas científicas entre estudantes do oitavo ano de uma escola urbana. Para tal, a partir do jogo School Scene Investigators: The Case of The Stolen Score Sheets [Bressler e Bodzin 2013, apud Bressler e Bodzin 2016], baseado em um mistério envolvendo ciência forense, foi desenvolvido um novo e mais amplo jogo, School Scene Investigators: The Case of The Mystery Powder, este último baseado em realidade aumentada, no qual os estudantes deveriam investigar um pó misterioso encontrado na caixa registradora da cafeteria da escola após a ocorrência de um roubo. Nesse sentido, os estudantes deveriam trabalhar em grupos, atuando colaborativamente na realização de uma série de tarefas para solucionar o mistério. Para isso, deveriam utilizar iPads para explorar códigos QR espalhados pela escola, que disponibilizavam conteúdos visuais em realidade aumentada. Os autores concluíram que um jogo educacional sério bem desenvolvido promove maior nível de engajamento e condução de práticas científicas em comparação com atividades de laboratório tradicionais, na medida em que os resultados obtidos pelos participantes do jogo nesses aspectos foram significativamente melhores que os obtidos pelo grupo controle.

[A4] No estudo, [Bressler et al. 2019] examinaram práticas discursivas de três equipes de estudantes em um jogo, com o objetivo de identificar conexões entre elementos do discurso colaborativo, estilos de linguagem e condução de práticas científicas. Nesse sentido, foi analisado o jogo School Scene Investigators: The Case of The Mystery Powder, mencionado em [A3], no qual conteúdos visuais em realidade aumentada são disponibilizados através da exploração de códigos QR espalhados pela escola. Através da realização de análise de rede, um método para analisar elementos em dados codificados e estabelecer conexões como modelos dinâmicos em rede epistêmica [Shaffer 2006; Shaffer 2017, apud Bressler et al. 2019], os autores concluíram que os estudantes desenvolveram práticas científicas durante o jogo. Além disso, houve engajamento na comunicação e foi identificado um estilo de linguagem comunal, no qual os estudantes dirigiam-se ao grupo coletivamente, utilizando elementos discursivos que se referiam à equipe, em lugar de referências a pessoas específicas.

[A5] Neste trabalho, [Chiang, Yang e Hwang 2014] destacam a importância do engajamento dos estudantes em atividades de construção colaborativa de conhecimento, e investigam práticas de compartilhamento de conhecimentos a partir da criação de um ambiente de realidade aumentada baseado em localização. Nesse sentido, foi conduzido um estudo em um curso de ciências naturais, cujos participantes eram estudantes do quarto ano do Ensino Fundamental de uma escola em Taiwan. Os autores concluíram que a atividade de investigação envolvendo realidade aumentada promoveu maior engajamento e maior nível de interação entre os estudantes para construção de conhecimentos.

[A6] No estudo, [Jesionkowska, Wild e Neval 2020] investigaram o método de aprendizagem ativa para o trabalho com conteúdos em STEAM. Nesse sentido, os estudantes deveriam construir um aplicativo baseado em realidade aumentada como parte do processo de aprendizagem. Participaram da pesquisa estudantes do Ensino Médio de uma escola em Oxford. Os autores apontaram que todos os participantes 
foram bem-sucedidos na criação dos aplicativos, alguns de grande complexidade. Além disso, mencionam que os estudantes declararam que as aulas envolvendo realidade aumentada eram mais agradáveis, e que tal tecnologia poderia contribuir no processo de aprendizagem.

[A7] Neste trabalho, [Litts, Lewis e Mortensen 2019] investigam que aspectos do pensamento computacional jovens aprendem desenvolvendo jogos para celular em suas comunidades. No estudo conduzido em uma cidade rural dos Estados Unidos, após a realização de seis workshops de duas horas cada, os participantes deveriam desenvolver jogos para celular baseados em localização para compartilhar experiências sobre questões ambientais ou cotidianas, através da utilização da plataforma de programação Augmented Reality and Interactive Storytelling (ARIS). Nesse sentido, foram desenvolvidos jogos que abordavam questões como incêndios florestais, adoção animal e poluição. Os resultados demonstraram como estudantes podem se engajar em questões que envolvem suas comunidades através de artefatos computacionais e práticas relacionadas com o pensamento computacional. Além disso, os autores concluíram que a proposta permite a condução de práticas interdisciplinares que contam com a participação de um público autêntico, a comunidade local, além de possibilitar uma participação equitativa em práticas de computação.

[A8] Neste trabalho, [Cai 2019] analisa o uso de três aplicativos para celular baseados em realidade aumentada em uma série de aulas de probabilidade em uma turma de Ensino Fundamental de uma escola chinesa, bem como as atitudes dos participantes na interação com os aplicativos. Os estudantes, cujas idades variavam entre 13 e 15 anos, foram divididos em um grupo experimental, no qual os participantes utilizaram os aplicativos, e um grupo controle, no qual foram empregadas atividades tradicionais. Os resultados apontaram que o uso dos aplicativos baseados em realidade aumentada foi positivo para a aprendizagem de tópicos de probabilidade, na medida em que observou-se, a partir de uma análise de covariância, que os participantes do grupo experimental obtiveram maiores ganhos em termos de aprendizagem que os estudantes do grupo controle.

[A9] No trabalho, [Bartholomew 2017] discorre sobre como jogos, aplicativos e novas tecnologias podem ser utilizados para ensinar conteúdos em STEM através de uma abordagem integrativa, incorporando o ensino de diferentes componentes curriculares por meio do envolvimento dos estudantes em atividades que conectam as diferentes áreas do conhecimento [Reeve 2015; Wells e Ernst 2015, apud Bartholomew 2017]. Mais especificamente, o autor aborda o uso do jogo Pokémon Go, derivado da franquia Pokémon, com finalidades pedagógicas. O jogo se baseia na utilização de tecnologia GPS de telefones celulares, de modo que enquanto a tela do aparelho apresenta a localização real do usuário, é possível encontrar Pokémons (monstros virtuais) em realidade aumentada, que podem ser capturados. O autor concluiu que existem diversas potencialidades de utilização do jogo relacionadas a conteúdos em STEM, tais como estudo de características e habitats de animais e plantas, construção de conhecimentos relacionados a softwares e à realidade aumentada, estudo de mapas, estudo de probabilidades, estatísticas e cálculo de distâncias, entre outros.

[A10] Neste estudo, [Wang et al. 2017] exploram os desenvolvimentos e usos recentes da realidade aumentada em contextos educacionais formais e informais. Nesse sentido, são apresentados estudos de casos ilustrando a implementação de realidade 
aumentada em diferentes abordagens pedagógicas, sejam elas de natureza instrucional, construcionista ou colaborativa. Os autores concluem que, nos diversos contextos educativos, e a partir de diferentes abordagens, a realidade aumentada tem a potencialidade de trazer grandes inovações nas formas de aprendizagem. Destaca-se, ainda, que ambientes de aprendizagem em realidade aumentada permitem que cada aluno experiente um caminho único de descoberta através da conexão entre aprendizagem, tecnologia e vida real. Além disso, possibilita-se o engajamento de estudantes da solução de problemas complexos e construção de um aprendizado mais profundo.

A leitura dos artigos possibilitou identificar uma série de potencialidades no uso de tecnologias envolvendo realidade aumentada na Educação Básica. Quanto às práticas pedagógicas exploradas, destacaram-se: visualização de microorganismos sem a utilização de microscópios, criação de jogos utilizando coordenadas GPS, aprendizagem baseada em investigação (inquiry-based learning), desenvolvimento do pensamento computacional através da criação de programas de computador envolvendo realidade aumentada, estudo de probabilidades, estudo de astronomia, uso do jogo Pokemon Go para exploração de conteúdos em STEM, estudo do funcionamento e programação de robôs e aprendizagem colaborativa remota. Tal fato se alinha com as propriedades destacadas por [Azuma 2001], na medida em que são combinados objetos reais e virtuais em um mesmo ambiente real e ocorre interatividade em tempo real.

A implementação de tecnologias digitais e interativas na Educação permite ampliar as possibilidades de aprendizagem dos alunos, uma vez que as ferramentas disponíveis são versáteis, adaptáveis e promovem novas experiências para aqueles que não se adaptam às metodologias tradicionais de ensino. Nesse sentido, a realidade aumentada permite que o aluno amplie seu horizonte de aprendizagem e mistura o mundo real com o mundo virtual [Macedo e Fernandes 2015]. Tais potencialidades de situações de aprendizagem que mesclem aspectos da realidade com elementos virtuais foram observadas em todos os artigos analisados. Além disso, na maior parte dos trabalhos analisados eram utilizados aplicativos já existentes de smartphones, ou os mesmos eram desenvolvidos pelos próprios estudantes, o que permite $o$ desenvolvimento de habilidades relacionadas com o pensamento computacional. Ademais, na maior parte dos estudos não foram utilizados equipamentos tecnológicos com alto grau de complexidade, o que possibilita que tais ferramentas sejam utilizadas em escolas com menos recursos disponíveis.

Por fim, outro fator apontado em diversos dos trabalhos analisados foi o impacto positivo das atividades envolvendo realidade aumentada no engajamento dos estudantes. Como tecnologias digitais fazem parte do cotidiano dos alunos, a utilização das mesmas em situações de aprendizagem aproxima as práticas pedagógicas da realidade do educando, o que contribui para o despertar da curiosidade e aumento da motivação. Além disso, pelo fato de tais atividades serem mais dinâmicas e, em geral, demandarem uma atitude colaborativa para a solução de problemas, é possível desenvolver habilidades de comunicação, socialização e trabalho em equipe, fato que foi amplamente observado nos artigos analisados. 


\section{Considerações finais}

As potencialidades pedagógicas de situações de aprendizagem envolvendo novas tecnologias são vastas e promissoras. Em virtude da flexibilidade de tais recursos, é possível desenvolver cenários que contemplem diferentes estilos de aprendizagem, contribuindo para aumentar o engajamento de estudantes com diferentes perfis nas atividades escolares. Os ganhos em motivação possibilitados pelo uso de tecnologias se relacionam com a inclusão, no âmbito educacional, de uma realidade que faz parte do cotidiano de uma geração que já nasceu em um contexto altamente tecnológico. Assim, a adoção de práticas pedagógicas que envolvem tais tecnologias, além de contribuir para a disseminação de novas formas de ensinar e aprender, possibilita a ampliação da curiosidade e do nível de participação dos estudantes nas atividades propostas.

A maior parte das produções analisadas neste estudo abordou situações de aprendizagem envolvendo jogos digitais que exploravam ambientes de realidade aumentada baseados em localização. Tal fato pode ser justificado pela possibilidade de criação de tais ambientes utilizando programas específicos que não demandam habilidades mais complexas de programação. Desse modo, é possível criar cenários a serem explorados pelos estudantes em uma perspectiva ativa, colocando em relevo uma metodologia de aprendizagem baseada em investigação (inquiry-based learning), que foi explorada em vários dos trabalhos analisados. Assim, estudantes se tornam protagonistas no processo de aprendizagem, reunindo informações disponibilizadas em um cenário que mescla aspectos da realidade com elementos virtuais. Com isso, podem construir conhecimentos através da exploração de elementos das instalações físicas da escola e de seus entornos, o que contribui, ainda, para um melhor conhecimento da realidade local.

Por outro lado, o uso de recursos tecnológicos na Educação como uma forma de favorecer os processos de ensino e aprendizagem ainda é objeto de discussão e controvérsias nos espaços escolares. Apesar de tais recursos estarem presentes no cotidiano das pessoas e serem utilizados em diversas áreas de conhecimento, ainda é necessário promover condições de acesso a tais recursos à comunidade escolar, bem como qualificação para utilização pelos professores. A incorporação das tecnologias no meio educacional e, principalmente, nas salas de aula, depende da coexistência de diversos fatores, como novas formas de pensar e fazer, disponibilidade dos próprios recursos tecnológicos e a disposição em aprender por meio de novos métodos. No que se refere ao contexto brasileiro, embora a Base Nacional Comum Curricular (BNCC) destaque a importância da utilização de novas tecnologias em situações de aprendizagem, o que se observa é que as limitações estruturais, em espacial em escolas públicas, impedem ou limitam esse tipo de abordagem. Notou-se, neste sentido, que nenhum dos artigos analisados nesta pesquisa foi produzido no contexto nacional. Ainda assim, existe grande potencial para utilização da tecnologia no campo educacional, mesmo que os recursos tecnológicos disponíveis sejam limitados. Como pôde-se observar nos artigos analisados, é possível construir jogos digitais e cenários imersivos em realidade aumentada com a utilização de recursos como smartphones, tecnologias de que muitos estudantes dispõem.

Por fim, associar Educação às tecnologias digitais e interativas pode ser um caminho promissor para tornar os processos educativos mais flexíveis, em que os aprendentes tenham ao seu alcance uma proposta pedagógica que contemple suas 
necessidades e que propicie experiências mais significativas, minimizando as distâncias entre a aprendizagem e a aplicabilidade dos conteúdos. Através da revisão de literatura, foi possível encontrar evidências de que aproximar as tecnologias digitais e interativas ao cotidiano das escolas na Educação Básica promove o engajamento dos estudantes, contribuindo para os processos de ensino e aprendizagem. Para trabalhos futuros, pretende-se analisar como artefatos voltados à realidade aumentada têm sido utilizados em situações de aprendizagem em outros contextos, como no Ensino Superior e na Educação Especial. Finalmente, é importante que professores sejam capazes de utilizar novas tecnologias cada vez mais em suas salas de aulas e nos demais espaços escolares, na medida em que elas representam poderosas ferramentas pedagógicas, com grande potencial de contribuição para estimular a aprendizagem dos alunos de forma inovadora. Cabe ressaltar, no entanto, que é essencial que as diferentes esferas governamentais atuem no sentido de promover recursos e infraestrutura adequada para a utilização de tais tecnologias no ambiente escolar.

\section{Referências}

Abdusselam, S. et al. (2018) "Examining microscopic organisms under augmented reality microscope: a 5E learning model lesson", Science Activities, 55:1-2, p. 68-74.

Annetta, L. et al. (2012) "Augmented technology games: using technology on a budget”, Science Scope, p. 26-32.

Azuma, R. et al. (2001) "Recent advances in augmented reality", IEEE Computer Graphics and Applications, vol. 21, p. 34-47.

Bartholomew, S. (2017) "Using Pokemon Go to teach integrative STEM", Technology and Engineering Teacher, 76(5), p. 24-27.

Bressler, D. e Bodzin, A. (2016) "Investigating flow experience and scientific practices during a mobile serious educational game", Journal of Science Education Technology, 25(5), p. 795-805.

Bressler, D. et al. (2019) "Using epistemic network analysis to examine discourse and scientific practice during a collaborative game", Journal of Science Education Technology, 28(5), 553-566.

Cai, S. et al. (2019) "Probability learning in mathematics using augmented reality: impact on student's learning gains and attitudes", Interactive Learning Environments, p. 1-14.

Chiang, T., Yang, S. e Hwang, G. (2014) "Students' online interactive patterns in augmented reality-based inquiry activities", Computers \& Education, 78, p.97-108.

Forte, C. e Kirner, C. (2009) "Usando Realidade Aumentada no Desenvolvimento de Ferramenta para Aprendizagem de Física e Matemática", Atas do $6^{\circ}$ Workshop de realidade virtual e aumentada, Santos.

Herrera, L., Pérez, J. e Ordóñez, S. (2019) "Developing spatial mathematical skills through 3D tools: augmented reality, virtual environments and 3D printing", International Journal on Interactive Design and Manufacturing (IJIDeM). 
Jesionkowska, J., Wild, F e Deval, Y. (2020) “Active learning augmented reality for STEAM education - a case study", Education Sciences, v. 10, n. 198.

Lee, K. (2012) “Augmented reality in education and training”, TechTrends, v. 56, n.2, mar/abr.

Litts, B., Lewis, W. e Mortensen, C. (2019) "Engaging youth in computational thinking practices through designing place-based mobile games about local issues", Interactive Learning Environments, 28(3), 302-315.

Macedo, S. e Fernandes, F. (2015) "Realidade aumentada e possibilidade de uso na educação", In: PEIXOTO et al. (org.). Tecnologias digitais na educação: pesquisas e práticas pedagógicas. Campos dos Goytacazes, RJ: Essentia, p. 95-105.

Silva, A. (2013) "Uso de recurso educacional com mídias interativas e integradas online em ensino e aprendizagem", 120f, Dissertação (Mestrado em Ensino de Ciências) - Universidade Federal de Itajubá, Itajubá.

Tori, R., Kirner, C e Siscoutto, R. (2006) "Fundamentos e tecnologia de realidade virtual e aumentada", Porto Alegre: Sociedade Brasileira de Computação, 422p.

Wang, M. et al. (2017) "Augmented reality in education and training: pedagogical approaches and illustrative case studies", J Ambient Intell Human Comput, 9, p. 1391-1402. 